\title{
BMJ Open Development of a patient decision aid on subacromial decompression surgery and rotator cuff repair surgery: an international mixed-methods study
}

\author{
Joshua Zadro (D , ${ }^{1}$ Caitlin Jones, ${ }^{1}$ Ian Harris, ${ }^{2}$ Rachelle Buchbinder, ${ }^{3}$ \\ Denise A O'Connor (D) ," Kirsten McCaffery (D) , ${ }^{5}$ Rachel Thompson, ${ }^{6}$ \\ Sascha Karunaratne (D) ," Min Jiat Teng (D) , ${ }^{1}$ Christopher Maher, ${ }^{1}$ \\ Tammy Hoffmann ${ }^{8}$
}

To cite: Zadro J, Jones C, Harris I, et al. Development of a patient decision aid on subacromial decompression surgery and rotator cuff repair surgery: an international mixedmethods study. BMJ Open 2021;11:e054032. doi:10.1136/ bmjopen-2021-054032

- Prepublication history and additional supplemental material for this paper are available online. To view these files, please visit the journal online (http://dx.doi.org/10.1136/ bmjopen-2021-054032).

Received 01 June 2021 Accepted 16 August 2021

Check for updates

(C) Author(s) (or their employer(s)) 2021. Re-use permitted under CC BY-NC. No commercial re-use. See rights and permissions. Published by BMJ.

For numbered affiliations see end of article.

Correspondence to

Dr Joshua Zadro;

joshua.zadro@sydney.edu.au

\section{ABSTRACT}

Objective To develop and user test a patient decision aid for people with subacromial pain syndrome that presents evidence-based information on the benefits and harms of subacromial decompression surgery and rotator cuff repair surgery.

Design Mixed-methods study outlining the development of a patient decision aid.

Setting We assembled a multidisciplinary steering group, and used existing decision aids and decision science to draft the decision aid. Participants were recruited through social media (not restricted by country nor setting), local hospitals and the authors' collaboration network.

Participants People with shoulder pain and health professionals who manage people with shoulder pain. Primary and secondary outcomes We interviewed participants to gather feedback on the decision aid, assessed useability and acceptability (using qualitative and quantitative methods) and performed iterative cycles of redrafting the decision aid and reinterviewing participants as necessary. Interview data were analysed using thematic analysis. Quantitative data were summarised descriptively.

Results We interviewed 26 health professionals (11 physiotherapists, 7 orthopaedic surgeons, 4 general practitioners, 3 chiropractors and 1 osteopath) and 14 people with shoulder pain. Most health professionals and people with shoulder pain rated all aspects of decision aid acceptability as adequate-to-excellent (eg, length, presentation, comprehensibility). Interviews highlighted agreement among health professionals and people with shoulder pain on most aspects of the decision aid (eg, treatment options, summary of benefits, harms and practical issues, questions to ask a health professional, graphics, formatting). However, some aspects of the decision aid elicited divergent views among health professionals (eg, causes and symptoms of shoulder pain, evidence on benefits and harms).

Conclusion This decision aid could be an acceptable and valuable tool for helping people with subacromial pain syndrome make informed treatment choices. A randomised controlled trial evaluating whether this decision aid reduces people's intentions to undergo shoulder surgery and facilitates informed treatment choices is underway.
Strengths and limitations of this study

- This is the first study to rigorously describe the development of a patient decision aid for people with subacromial pain syndrome that presents evidencebased information on the benefits and harms of subacromial decompression surgery and rotator cuff repair surgery, compared with non-surgical options.

- We developed the patient decision aid with guidance from the International Patient Decision Aids Standards, used a mixed-methods approach to evaluate useability and acceptability, interviewed a broad range of health professionals and patients, and conducted one-on-one interviews which allowed in-depth feedback on the decision aid.

- Our decision aid includes several key features recommended to optimise risk communication (eg, presenting numeric estimates, presenting uncertainty, using visuals, tailoring estimates).

- Limitations include a small sample size for our quantitative acceptability data, being unable to recruit certain groups of health professionals (eg, rheumatologists, sports doctors) and only interviewing people who speak English.

Trial registration number ACTRN12621000992808

\section{INTRODUCTION}

Subacromial decompression surgery and rotator cuff repair surgery (with or without decompression) are frequently performed for people with subacromial pain syndrome $\mathrm{e}^{1-4}$ an umbrella diagnosis that accounts for $85 \%$ of cases of shoulder pain (including rotator cuff tears)-but evidence suggests these procedures provide limited clinical benefit. Subacromial decompression surgery is not superior to placebo (high-certainty evidence) or non-surgical options, such as exercise and glucocorticoid injections (low-certainty to 
moderate-certainty evidence), for improving pain and function in people with subacromial pain syndrome. ${ }^{5}$ Rotator cuff repair surgery is not superior to non-surgical options for degenerative rotator cuff tears (low-certainty to moderate-certainty evidence) ${ }^{6}$ Serious harms (eg, infection) are experienced by $6 / 1000$ people who have arthroscopic shoulder surgery. ${ }^{5}$

Use of subacromial decompression surgery and rotator cuff repair surgery is increasing globally ${ }^{1-4}$ despite the above evidence, suggesting people may not be making informed treatment choices. In Australia, the annual number of subacromial decompression surgeries performed increased from 3536 to 7455 between 2000 and 2019 , while the number of rotator cuff repair surgeries performed increased from 6212 to 12436 during this period. ${ }^{1}$ Increases have also been reported in the USA, ${ }^{4}$ England ${ }^{27}$ and Finland. ${ }^{3}$

Patient decision aids present unbiased information on the benefits and harms of different healthcare options. A decision aid on options for treating subacromial pain syndrome could help patients make informed treatment choices and result in less use of unnecessary surgery. A Cochrane review of 105 studies $(n=31043)$ found that people exposed to decision aids made more informed choices about their healthcare and had a more active role in decision making, with no negative effects on outcomes or satisfaction. ${ }^{8}$ For some conditions, patients were also more likely to choose less invasive treatment options. ${ }^{8}$

By eliciting views of key stakeholders using mixed methods, our aim was to develop a patient decision aid for people with subacromial pain syndrome that presents evidence-based information on the benefits and harms of subacromial decompression surgery and rotator cuff repair surgery for subacromial pain syndrome (compared with non-surgical options).

\section{METHODS}

\section{Initial decision aid design}

We developed a patient decision aid with guidance from the International Patient Decision Aids Standards (IPDAS) using mixed methods. ${ }^{9} 10$ We began by assembling a multidisciplinary steering group (study authors) including topic experts (IH: orthopaedic surgery; RB: shoulder pain; KM, TH, RET and DAO'C: patient decision aids and shared decision making) and health professionals who manage people with shoulder pain (JZ and SK: physiotherapists; RB: rheumatologist). The first draft of the decision aid was created in PowerPoint and based on decision aids for antibiotics ${ }^{11}$ and knee arthroscopy $^{12}$ which several study authors have developed (TH, $\mathrm{KM}, \mathrm{RB}, \mathrm{DAO} \mathrm{C}^{\mathrm{C}}$ and $\mathrm{IH}$ ) (online supplemental file 1). Key features adapted from these decision aids included horizontal bar graphs displaying the effects of surgery compared with placebo and non-surgical options (which included injections, physiotherapy, medication and wait and see), icon arrays to help patients understand probabilities, a statement about the source and quality of the evidence, questions for patients to ask their health professional and practical issues (eg, time off work, driving restrictions). Decision science evidence suggests these features improve patient decision making. ${ }^{13-17}$ Data from the 2019 Cochrane reviews on subacromial decompression surgery ${ }^{5}$ and rotator cuff repair surgery ${ }^{6}$ were used to inform numeric estimates of benefits and harms used in the decision aid. Expert opinion and consensus from the steering group was used to inform all information presented in the decision aid (eg, causes and symptoms of shoulder pain, practical issues). The steering group provided feedback on the first draft before we conducted semistructured interviews with people with shoulder pain and health professionals who manage people with shoulder pain.

\section{Participants}

Twenty-six health professionals involved in the management of shoulder pain were recruited through social media, Royal Prince Alfred and Concord Hospitals in Sydney (Australia), and the study authors' collaboration network. Health professionals had to manage/consult at least five people with suspected subacromial pain syndrome per year. There was no restriction on the type of health professional (eg, orthopaedic surgeon, physiotherapist, general practitioner (GP)), work setting or country of practice, or years of experience. Fourteen people with self-reported shoulder pain (hereafter referred to as 'patients') were recruited through social media and referrals from health professionals who participated in the study. Patients had to be $\geq 18$ years old and able to understand and communicate in English to participate. There was no restriction on their country of birth. Enrolled participants were asked if they had any contacts who met our inclusion criteria (snowballing). We purposively sampled participants to achieve diversity in age, gender and ethnicity. For health professionals, we also purposively sampled to achieve diversity in profession, years of experience and country of practice. All participants provided consent by checking a box before proceeding to the preinterview online questionnaire that confirmed they had read the participants information sheet and consent form and agree to participate in the study.

\section{Data collection}

We reported the qualitative aspect of this study according to the 32-item Consolidated Criteria for Reporting Qualitative Research checklist (online supplemental file 2). ${ }^{18}$ Box 1 describes the data collection process including the preinterview questionnaires (used to purposively sample participants; online supplemental files 3 and 4), semistructured interviews (topic guides in online supplemental files 5 and 6 ) and acceptability questionnaires (online supplemental files 7 and 8). In accordance with IPDAS guidance, ${ }^{9}{ }^{10}$ semistructured interviews were used to assess patients' views on decisional needs and health professionals' views on patients' decisional needs, gather feedback on the draft decision aid, and assess useability and acceptability of the decision aid. Participants 


\section{Box 1 Data collection process}

\section{Preinterview questionnaires used to purposively sample participants}

For health professionals, we gathered data on demographics, profession, years of experience, clinical setting and number of patients with subacromial pain syndrome seen per year (online supplemental file 3). For patients, we gathered data on demographics (eg, age, gender), duration and severity of shoulder pain and previous treatments, previous imaging and previous sick leave for shoulder pain (online supplemental file 4).

\section{Semistructured interviews}

Interviews were used to gather feedback on the best way to present different aspects of the decision aid, such as treatment options, numeric estimates of benefits and harms, practical issues and questions to ask a health professional. Participants were then asked to 'think out loud' while they read through the decision aid. They were encouraged to say everything that came to mind (eg, concepts that might be challenging to understand, what their eye was drawn to) and give feedback on how the decision aid could be improved. The researcher conducting the interview used additional questions to prompt participants who were unsure of what to say. For example, some participants were prompted to give feedback on the relevance, usefulness, formatting, and language of each section, and the use of images. Interview guides for health professionals and patients are in online supplemental files 5 and 6 , respectively.

\section{Acceptability questionnaires}

After the first round of interviews ( $n=12$ health professionals; $n=7$ patients) and several redrafts, we began assessing acceptability with a brief questionnaire at the end of each interview because we felt we were getting close to the final version of the decision aid. A separate questionnaire, adapted from The Ottawa Hospital Research Institute, ${ }^{29}$ was used for health professionals (online supplemental file 7) and patients (online supplemental file 8).

were provided the draft decision aid prior to the interview but some participants did not review it beforehand. At the end of each interview, participants were given the opportunity to provide any additional feedback or comments. Changes to the decision aid were made throughout the interview process. Modifications were compared with older versions of the decision aid to understand whether changes were useful.

All interviews were conducted one-on-one via videoconference due to COVID-19. All interviews lasted between 30 and $60 \mathrm{~min}$ and were conducted by a researcher with experience in conducting qualitative interviews $(\mathrm{CJ})$. The interviewer was a female $\mathrm{PhD}$ candidate and occupational therapist. Two pilot interviews were conducted before recruitment to test the interview guides. During participant interviews, the interviewer took notes to highlight key concepts emerging from the interview and direct further questioning. The interviewer did not have an established relationship with participants prior to the study commencing. Participants were informed of the reason for the study prior to being interviewed. All interviews were audiorecorded (with verbal consent obtained from participants) and transcribed verbatim for analysis. All participants had the opportunity to review the transcript of their interview prior to data analysis if they wished. Health professionals and patients and who completed an interview were compensated for their time with a US\$100 and US\$50 supermarket gift card, respectively. Health professionals were compensated with more money to account for potentially sacrificing appointment slots to participate in this study.

\section{Data analysis}

Preinterview and acceptability questionnaire responses were summarised using descriptive statistics (means and SDs, counts and percentages). For the health professional acceptability questionnaire (online supplemental file 7), a five-point Likert scale (strongly agree $=5$; strongly disagree $=1$ ) was used to assess agreement with various statements. We presented Likert scores as the percentage of responses for each category and as means (SD). We also calculated mean (SD) agreement scores for orthopaedic surgeons separately as we anticipated they might have different views on a decision aid for people considering surgery compared with other health professionals. For the patient acceptability questionnaire (online supplemental file 8), impressions of different sections of the decision aid were dichotomised as 'excellent/ good' versus 'fair/poor'.

All interview data were analysed using thematic analysis; a method for identifying, analysing and reporting patterns within data. ${ }^{19}$ Grounded theory using an inductive approach underpinned how data was collected and analysed. Two researchers $(\mathrm{CJ}$ and JZ) independently familiarised themselves with the interviews (via audiorecordings or transcripts), recorded initial observations and identified concepts relevant to the questions asked. The two researchers developed a framework to organise concepts into broader themes and subthemes in Excel. ${ }^{20}$ Any disagreements in categorising concepts into themes and subthemes were discussed and resolved. The mapping of themes and subthemes was iterative as new data emerged so that the decision aid was continually updated before new interviews were conducted. Over 10 iterative cycles of revisions were performed. However, in some cases these were very minor changes (eg, correcting typos, rewording a sentence). Patients' views on decisional needs and health professionals' views on patients' decisional needs were integrated with the feedback given on each section of the decision aid to streamline the presentation of the results. Interviews stopped once no new feedback was being provided (data saturation) and participants had an overall positive impression of the decision aid.

\section{Patient or public involvement}

Patients and members of the public were not involved in the design of this study.

\section{RESULTS}

\section{Adherence to the IPDAS criteria and user-centredness}

We determined that the decision aid (online supplemental file 9) met 6 out of 6 criteria to be considered a decision aid, 6 out of 6 criteria to reduce the risk of 
harmful bias, and 20 and 23 quality criteria according to the IPDASi checklist (V.4.0) ${ }^{21}$ (online supplemental file 10). Our decision aid also met 10 out of 11 criteria for user-centredness (online supplemental file 11), as assessed by the User-Centred Design 11-item measure. ${ }^{22}$

\section{Participant characteristics and decision aid acceptability}

We interviewed 26 health professionals (11 (42\%) physiotherapists, $7(27 \%)$ orthopaedic surgeons, $4(15 \%)$ GPs, 3 (12\%) chiropractors and $1(4 \%)$ osteopath) and 14 patients. Repeat interviews were conducted with one of these health professionals (physiotherapist) and four of these patients to explore whether initial feedback had been addressed through modifications to the decision aid. No participant who completed the preinterview questionnaire refused an interview. However, a number of participants who completed the preinterview questionnaire were not interviewed since participants were purposively sampled $(n=130$ health professional and $n=19$ patient respondents were not interviewed). Health professional and patient characteristics are in table 1 . There were 15 health professionals and 11 patients who completed the acceptability questionnaire. All aspects of decision aid acceptability were rated as adequate-to-excellent (eg, length, amount of information, presentation, comprehensibility) by most health professionals (table 2) and patients (table 3). Figure 1 provides a summary of the development process.

\section{Feedback on each section of the decision aid}

Positive feedback for each section, and for the decision aid overall, largely included agreement with the content, graphics, formatting, amount of information and presentation of information. Online supplemental file 12 provides a summary of themes and subthemes across sections of the decision aid. Suggestions for improvement (themes) and examples (subthemes) are summarised below. Although most suggestions were implemented, some conflicted with others or were not possible to implement. Online supplemental file 13 outlines feedback we did not incorporate in the decision aid and our justification for this. Feedback from three or more types of health professionals was classified as 'multidisciplinary feedback'.

\section{Who should read this decision aid?}

This section covers the title of the decision aid, information about who should read the decision aid, and common causes and symptoms of shoulder pain. Suggestions for improvement (themes) with examples (subthemes) included:

- Improve clarity on the target population (eg, some GPs wanted this section to be more concise, some patients thought softening the exclusion criteria would prevent people with overlapping symptoms disregarding the decision aid).

- Highlight that patients need to discuss this decision aid with a health professional (multidisciplinary feedback).
Table 1 Characteristics of health professionals who manage people with shoulder pain $(n=26)$ and people with shoulder pain $(n=14)$

\begin{tabular}{|c|c|}
\hline Health professionals & $\begin{array}{l}\text { Mean (SD) or N (\%) } \\
\text { (unless specified } \\
\text { otherwise) }\end{array}$ \\
\hline \multicolumn{2}{|l|}{ Profession } \\
\hline Physiotherapist & $11(42)$ \\
\hline Orthopaedic surgeon & $7(27)$ \\
\hline General practitioner & $4(15)$ \\
\hline Chiropractor & $3(12)$ \\
\hline Osteopath & $1(4)$ \\
\hline Age (years) & $40(11)$ \\
\hline Female & $8(31)$ \\
\hline \multicolumn{2}{|l|}{ Country of practice } \\
\hline Australia & $18(69)$ \\
\hline USA & $4(15)$ \\
\hline Canada & $2(8)$ \\
\hline England & $2(8)$ \\
\hline Years of experience & $12(9)$ \\
\hline Works in private practice & $19(73)$ \\
\hline $\begin{array}{l}\text { No of patients with shoulder pain seen per } \\
\text { year }\end{array}$ & $\begin{array}{l}164(167) \\
\text { Median (IQR): } 100 \\
(40-250)\end{array}$ \\
\hline People with shoulder pain & $\begin{array}{l}\text { Mean (SD) or N (\%) } \\
\text { (unless specified } \\
\text { otherwise) }\end{array}$ \\
\hline Age (years) & $46(18)$ \\
\hline Female & $6(43)$ \\
\hline \multicolumn{2}{|l|}{ Highest level of education } \\
\hline University & $6(43)$ \\
\hline High school or TAFE/trade & $8(57)$ \\
\hline \multicolumn{2}{|l|}{ Country of birth } \\
\hline Australia & $10(71)$ \\
\hline Philippines & $1(7)$ \\
\hline USA & $1(7)$ \\
\hline UK & $1(7)$ \\
\hline Egypt & $1(7)$ \\
\hline \multicolumn{2}{|l|}{ Employment status } \\
\hline Working & $9(64)$ \\
\hline Not working & $3(21)$ \\
\hline Retired/unable to work & $2(14)$ \\
\hline Health insurance & $8(57)$ \\
\hline Duration of shoulder pain (months) & $\begin{array}{l}96(117) \\
\text { Median (IQR): } 18(6-180)\end{array}$ \\
\hline \multicolumn{2}{|l|}{ Activity interference in the past week } \\
\hline Not at all & $3(21)$ \\
\hline A little bit & $3(21)$ \\
\hline Moderately & $6(43)$ \\
\hline Quite a bit & $1(7)$ \\
\hline Extremely & $1(7)$ \\
\hline \multicolumn{2}{|l|}{ Management strategies trialled } \\
\hline Exercise & $9(64)$ \\
\hline
\end{tabular}

Continued 


\begin{tabular}{ll}
\hline Table 1 Continued & $\begin{array}{l}\text { Mean (SD) or N (\%) } \\
\text { (unless specified } \\
\text { otherwise) }\end{array}$ \\
\hline Health professionals & $8(57)$ \\
\hline Medication & $7(50)$ \\
\hline Rest & $6(43)$ \\
\hline Massage & $5(36)$ \\
\hline Manual therapy & $2(14)$ \\
\hline Injections & $2(14)$ \\
\hline $\begin{array}{l}\text { Surgery } \\
\text { Other }\end{array}$ & $3(21)$ \\
\hline $\begin{array}{l}\text { Previously had a scan (X-ray, MRI, } \\
\text { ultrasound) }\end{array}$ & $8(57)$ \\
\hline $\begin{array}{l}\text { Previously had sick leave due to shoulder } \\
\text { pain }\end{array}$ & $2(14)$ \\
\hline
\end{tabular}

TAFE, Technical and Further Education.

- Revise the causes and symptoms of shoulder pain (eg, multidisciplinary feedback suggested this information had a pathoanatomical focus that was inaccurate and that this information could drive patients towards surgery).

- Use positive messaging (eg, some physiotherapists thought the language would cause fear among patients).

- Make this section more concise and relevant (eg, multidisciplinary feedback suggested the explanation of shoulder symptoms might be irrelevant for patients, some orthopaedic surgeons wanted to emphasise the importance of a proper diagnosis to guide treatment decisions).

Online supplemental file 14 highlights the changes between the first and final draft of this section.

\section{What are the treatment options covered in this decision aid?}

This section outlines non-surgical and surgical management options for subacromial pain syndrome. Suggestions for improvement included:

- Include more detail on non-surgical options and how to progress management (eg, multidisciplinary feedback suggested balancing the amount of information between the non-surgical and surgical options, some patients wanted more information on 'wait and see' and how to modify activities).

- Change the non-surgical options presented (eg, some physiotherapists thought it was inappropriate to include medication and injections as options, some physiotherapists and chiropractors thought the order of non-surgical options might be inappropriate).

- Include indications for surgery (eg, multidisciplinary feedback suggested the inclusion of indicators for each surgery like failed conservative management, severe pain, age and massive cuff tears).

- Present evidence of benefits and harms in this section (eg, multidisciplinary feedback suggested mentioning the success rate of surgery and non-surgical options and emphasise the harms of surgery).

- Change the information on surgery (eg, some patients wanted more detail on surgery and rehabilitation, while others wanted less detail on the procedures).

- Modify the formatting and graphics (eg, multidisciplinary feedback suggested listing non-surgical options first, some patients wanted more space between the options and thought the image of surgery was too graphic).

Online supplemental file 15 highlights the changes between the first and final draft of this section.

What are the likely benefits of surgery compared with non-surgical options?

This section summarises data on the effectiveness of subacromial decompression surgery and rotator cuff repair surgery compared with non-surgical options from two Cochrane reviews. ${ }^{5}{ }^{6}$ Suggestions for improvement included:

- Revise the description for the certainty of evidence (eg, some physiotherapists and chiropractors thought using a green font for high-certainty evidence would drive patients towards surgery).

- Evidence does not match experience, more clarification needed (eg, some orthopaedic surgeons thought the evidence from Cochrane reviews may not be generalisable, surgery may improve the speed of recovery and surgery may be useful for preventing tears progressing even if there was no improvement in symptoms, some orthopaedic surgeons and GPs thought it was important to acknowledge evidence represents averages and careful selection of surgical candidates could yield positive results).

- Simplify the statistics (eg, some physiotherapists and chiropractors thought 'key messages' could be used instead of a bar graph, some orthopaedic surgeons thought repetition of statistics was unnecessary and biased against surgery).

- Provide more detail or revise the description of the evidence (eg, some patients wanted information on the source of the evidence and more explanation about the certainty of evidence).

- Contextualise the evidence to reflect uncertainty on an individual level (eg, some patients wanted to highlight the numeric estimates were averages).

- Modify the formatting and language used (eg, some GPs and patients wanted to shorten the key messages box and include other information as footnotes, some patients thought the icon array was not useful).

Online supplemental file 16 highlights changes between the first and final draft of this section.

What are the likely harms of surgery?

This section summarises data on the potential harms of subacromial decompression and rotator cuff repair surgery from two Cochrane reviews. ${ }^{5}$ Data on the 




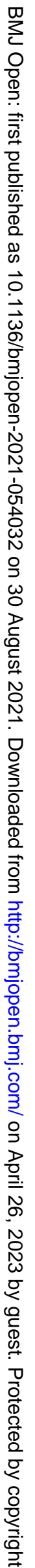


Table 3 Acceptability questionnaire for people with shoulder pain $(n=11)$

\begin{tabular}{|c|c|}
\hline Acceptability items & $\mathbf{N}(\%)$ \\
\hline \multicolumn{2}{|l|}{ Information presented was 'excellent or good'* } \\
\hline Subacromial shoulder pain: should I have surgery? & $9(82)$ \\
\hline Causes and symptoms of subacromial shoulder pain & $8(73)$ \\
\hline $\begin{array}{l}\text { What are the treatment options covered in this decision } \\
\text { aid? (Non-surgical options) }\end{array}$ & $10(91)$ \\
\hline $\begin{array}{l}\text { What are the treatment options covered in this decision } \\
\text { aid? (Surgery) }\end{array}$ & $9(82)$ \\
\hline $\begin{array}{l}\text { What are the likely benefits of surgery and non-surgical } \\
\text { options? }\end{array}$ & $9(82)$ \\
\hline What are the likely risks of surgery? & $8(73)$ \\
\hline What practical issues should I consider? & $10(91)$ \\
\hline $\begin{array}{l}\text { Questions to consider when talking with your health } \\
\text { professional }\end{array}$ & $10(91)$ \\
\hline \multicolumn{2}{|l|}{ Length of the decision aid } \\
\hline Just right & $8(73)$ \\
\hline Too short & $1(9)$ \\
\hline Too long & $2(18)$ \\
\hline \multicolumn{2}{|l|}{ Amount of information } \\
\hline Just right & $10(91)$ \\
\hline Too little information & $0(0)$ \\
\hline Too much information & $1(9)$ \\
\hline \multicolumn{2}{|l|}{ Presentation } \\
\hline Balanced & $2(18)$ \\
\hline Slanted towards surgery & $1(9)$ \\
\hline Slanted towards non-surgical options & $8(73)$ \\
\hline Useful when deciding about surgery & $11(100)$ \\
\hline Makes decision to have surgery easier & $8(73)$ \\
\hline Enough information provided & $9(82)$ \\
\hline
\end{tabular}

${ }^{*}$ Compared with 'fair/poor'. potential harms of non-surgical options were not available. Suggestions for improvement included:

- Present both minor and serious harms (multidisciplinary feedback).

- Provide more context for harms (eg, some physiotherapists and chiropractors suggested comparing the harms of surgery and non-surgical options, some GPs and patients thought presenting harms in a different section to 'benefits' does not give an understanding of harm vs benefit).

- Clarify the evidence as it does not match personal experience (eg, some orthopaedic surgeons thought harms were overestimated, some physiotherapists thought harms were underestimated).

- Modify the formatting and language used (eg, some orthopaedic surgeons and patients thought 'harm' was too negative and suggested replacing it with 'risk').

Online supplemental file 17 highlights the changes between the first and final draft of this section.

Summary of benefits, harms and other practical issues

This section provides a summary of the benefits, harms and important practical issues of surgery and non-surgical options. Suggestions for improvement included:

- Revise information on costs (eg, some physiotherapists and GPs wanted specific cost information on surgery, some orthopaedic surgeons wanted to soften the language emphasising the costs of surgery, some chiropractors and patients wanted information on the costs of non-surgical options).

- Revise information on activity restrictions and postsurgical management (eg, some physiotherapists and orthopaedic surgeons suggested alternative time frames for postsurgery activity restrictions, some GPs wanted to emphasise symptoms may improve with or without surgery).

- Modify the formatting or language used (eg, some GPs and patients wanted to separate the practical issues by type of surgery, while some physiotherapists thought this would result in too much information).

Online supplemental file 18 highlights the changes between the first and final draft of this section.

\section{Questions to consider when talking with a health professional}

This section outlines questions patients should consider asking their health professional before deciding to have surgery. Suggestions for improvement included:

- Adding questions (eg, some physiotherapists suggested 'How long should I wait before considering surgery?').

- Removing questions (eg, some orthopaedic surgeons suggested removing 'Do I know enough about my condition' and 'Have I considered my individual circumstances?’).

- Modifying the formatting (eg, some physiotherapists wanted the heading to be inclusive of any health

Figure 1 Flow chart of the development process. 
professional while others thought these questions were better suited to GPs).

An early version of the decision aid included a section on 'Are there other things I can do?' Suggestions included activity modification, strength and endurance exercises, seeking advice from a health professional, and considering surgery if these options do not help. We received positive feedback from patients on this section and helpful suggestions from health professionals to add information to help people try non-surgical options first. However, we decided to remove this section to save space so we could provide more detail about non-surgical options on the first page.

Online supplemental file 19 highlights the changes between the first and final draft of this section.

\section{Overall feedback}

Overall feedback included:

- Reduce the amount of information (eg, multidisciplinary feedback suggested a two-page decision aid was ideal, some physiotherapists and orthopaedic surgeons suggested removing the question-asking section and the references).

- More detail needed (eg, some GPs wanted information on imaging and the importance of not missing a serious disease, some patients thought the last page lacked a solution if someone had tried everything).

- Formatting and distribution suggestions (eg, multidisciplinary feedback and feedback from patients suggested separate decision aids for each surgery was needed, some GPs wanted separate decision aids for surgical and non-surgical options, some physiotherapists and chiropractors suggested making a video summary of the decision aid, some physiotherapists and orthopaedic surgeons suggested the decision aid should be provided in clinics, early during treatment, when patients are considering surgery and/or after a patient received a diagnosis, some patients suggested emphasising the question-asking section).

Some orthopaedic surgeons felt the decision aid was not balanced and biased against surgery. Most patients stated that the decision aid had swayed them away from surgery. One patient was initially sway towards surgery after reading the decision aid-to have surgery before the risk of complications increased or pain got worse-but changed their mind after reviewing the decision aid in a repeat interview due to lack of evidence of benefit.

\section{DISCUSSION}

\section{Summary of findings}

Most health professionals and people with shoulder pain rated all aspects of decision aid acceptability as adequateto-excellent (eg, length, amount of information, presentation, comprehensibility). Interviews highlighted agreement with most aspects of the decision aid (eg, treatment options, summary of benefits, harms and practical issues, questions to ask a health professional, graphics, formatting, amount of information and presentation of information) and some divergent views among health professionals on parts of the decision aid (eg, causes and symptoms of shoulder pain, evidence on benefits and harms). To understand whether this tool adds value to clinical practice, a randomised controlled trial evaluating whether this decision aid reduces people's intentions to undergo shoulder surgery and facilitates informed treatment choices is underway.

\section{Strengths and limitations of this study}

We developed a decision aid according to the IPDAS criteria, used a mixed-methods approach to evaluate useability and acceptability, interviewed a broad range of health professionals and patients, and conducted oneon-one interviews which allowed in-depth feedback on the decision aid. Our decision aid includes several key features recommended to optimise risk communication (eg, presenting numeric estimates, presenting uncertainty, using visuals, tailoring estimates). ${ }^{17}$ Limitations include a small sample size for our quantitative acceptability data, being unable to recruit certain groups of health professionals (eg, rheumatologists, sports doctors) and the decision aid only being developed in English (the steering group will consider cross-cultural adaptation of this tool following its evaluation in a clinical trial). We also acknowledge that individual circumstances may limit the applicability of the evidence presented in the decision aid (eg, age, pain severity, activity levels, job demands, insurance coverage, caring responsibilities, involvement in sport).

\section{Meaning of the study}

Interviews highlighted high levels of agreement with most aspects of the decision aid among health professionals and patients, although we did find some divergent views among health professionals on parts of the decision aid. Highly consistent feedback included praise for including practical issues for surgery and non-surgical options and a global summary of the benefits and harms of each, praise for including questions to ask a health professional, and a comment that a two-page decision aid would be ideal if it included all information from the three-page version. We attempted to create a two-page version of the decision aid but were not able to do so without comprising useability and acceptability or removing important information.

Health professionals and patients largely agreed with the presentation of non-surgical and surgical options, with some patients pleased to have 'wait and see' included as this aligned with their experience of pain that has resolved without treatment. Most health professionals and patients wanted non-surgical options listed before surgery to mimic treatment recommendations in real life. However, evidence suggests people are more likely to think a decision aid is balanced if options are listed side-by-side. ${ }^{13}$ We listed the options side-by-side, with nonsurgical options on the left ('first'), as a compromise. 
A few physiotherapists thought it was inappropriate to include medication and injections as options and wanted physiotherapy-delivered treatments listed earlier. Cochrane reviews on treatments for subacromial pain syndrome show glucocorticoid injections are superior to placebo and provide similar effects to non-steroidal antiinflammatory drugs ${ }^{23}$ and physiotherapy-delivered treatments (eg, exercise, manual therapy, electrotherapy). ${ }^{24} 25$ There is no evidence physiotherapy-delivered treatments are superior to placebo. ${ }^{2425}$ For these reasons, we did not action their suggestions.

We found quite varied feedback on the causes and symptoms of shoulder pain and presentation of benefits. Most health professionals and patients thought the causes and symptoms of shoulder pain were accurate and easy to understand. However, some health professionals (mostly physiotherapists) thought the pathoanatomical description of shoulder pain was inappropriate and used language that could cause fear and drive patients towards surgery. Some health professionals and patients thought the icon array and bar graphs were helpful, which is consistent with evidence suggesting these graphics help people make value-aligned decisions. ${ }^{14}$ However, we replaced some icon arrays and bar graphs with a 'key messages' box to address feedback that the statistics needed to be simplified and less repetitive, and because 'fact boxes' are useful risk-communicating tools. ${ }^{26} \mathrm{We}$ kept numeric estimates in the key messages box due to evidence suggesting patients prefer numeric estimates over narrative descriptions of effect sizes (eg, 'small' effects). ${ }^{27}$

Some orthopaedic surgeons disagreed with evidence from Cochrane systematic reviews and thought the decision aid was biased against surgery. Some believed that, if surgeons selected surgical candidates carefully, surgery could improve the speed of recovery and prevent tears progressing (outcomes not assessed in Cochrane reviews), while minimising the risk of harm. On the other extreme were some physiotherapists, who suggested that Cochrane systematic reviews have underestimated the true harms of surgery. We did not change the evidence presented because it is vital numeric estimates of benefits and harms in decision aids are based on the highest quality available evidence. ${ }^{1528}$

Nearly three in four patients thought the decision aid was biased against surgery (table 3), likely because the evidence we presented shows subacromial decompression surgery and rotator cuff repair surgery are not superior to non-surgical management. ${ }^{56}$ This suggests tools for assessing perceived balance of decision aids may not be suitable when a decision aid presents information that counters prevailing norms.

We included health professionals practising in various counties to maximise the acceptability of this tool globally. As such, some information had to be made more general to accommodate the characteristics of different health systems. For example, we could not be specific about the costs of surgery or non-surgical options as this varies between countries due to factors like health system and insurance coverage. We also received feedback to mention physiotherapists as providers of injections as this is within the scope of some advanced practice physiotherapists in the UK.

\section{Implications for future research}

We are currently evaluating a print/online version of the decision aid in a randomised controlled trial including people with shoulder pain considering shoulder surgery. However, feedback from health professionals raised the possibility of future trials evaluating different formats of the decision aid (eg, video summary, decision aid specific to one shoulder surgery) in different populations (eg, patients who have consulted with a surgeon and know what surgery they are likely to receive).

\section{CONCLUSION}

By eliciting views of key stakeholders, we developed a patient decision aid that presents evidence-based information on the benefits and harms of subacromial decompression surgery, rotator cuff repair surgery and non-operative treatments for subacromial pain syndrome. Acceptability testing and interviews with health professionals and people with shoulder pain highlights this decision aid could be an acceptable and valuable tool for helping people with shoulder pain make informed treatment choices. A randomised controlled trial evaluating whether this decision aid reduces people's intentions to undergo shoulder surgery and facilitates informed treatment choices is underway.

\section{Author affiliations}

${ }^{1}$ Institute for Musculoskeletal Health, Sydney School of Public Health, University of Sydney and Sydney Local Health District, Camperdown, New South Wales, Australia ${ }^{2}$ Ingham Institute for Applied Medical Research, South Western Sydney Clinical School, UNSW, Sydney, New South Wales, Australia

${ }^{3}$ Dept of Epidemiology and Preventive Medicine, Monash University, Melbourne, Victoria, Australia

${ }^{4}$ School of Public Health and Preventive Medicine, Monash University, Melbourne, Victoria, Australia

${ }^{5}$ Sydney Health Literacy Lab, School of Public Health, The University of Sydney, Sydney, New South Wales, Australia

${ }^{6}$ School of Public Health, University of Sydney, Camperdown, New South Wales, Australia

${ }^{7}$ Surgical Outcomes Research Centre (SOuRCe), Royal Prince Alfred Hospital, Camperdown, New South Wales, Australia

${ }^{8}$ Institute for Evidence-Based Healthcare, Faculty of Health Sciences and Medicine, Bond University, Gold Coast, Queensland, Australia

Correction notice Rachel Elizabeth Thompson has been corrected to Rachel Thompson.

Twitter Joshua Zadro @zadro_josh and Rachel Thompson @rachelthomp

Contributors All authors critically revised the manuscript for important intellectual content and approved the final manuscript. Please find below a detailed description of the role of each author: JZ: conception and design, analysis and interpretation of data, drafting and revision of the manuscript, and final approval of the version to be published. CJ: conception and design, analysis and interpretation of data, drafting and revision of the manuscript and final approval of the version to be published. IH: conception and design, interpretation of data, drafting and revision of the manuscript and final approval of the version to be published. RB: conception and design, interpretation of data, drafting and revision of the manuscript and final approval of the version to be published. DAO'C: conception and design, 
interpretation of data, drafting and revision of the manuscript and final approval of the version to be published. KM: conception and design, interpretation of data, drafting and revision of the manuscript and final approval of the version to be published. RET: conception and design, interpretation of data, drafting and revision of the manuscript and final approval of the version to be published. SK: conception and design, interpretation of data, drafting and revision of the manuscript and final approval of the version to be published. MJT: conception and design, interpretation of data, drafting and revision of the manuscript and final approval of the version to be published. CM: conception and design, interpretation of data, drafting and revision of the manuscript and final approval of the version to be published. TH: conception and design, interpretation of data, drafting and revision of the manuscript and final approval of the version to be published. JZ attests that all listed authors meet authorship criteria and that no others meeting the criteria have been omitted.

Funding This study was funded by the Arthritis Australia Grants-in-Aid scheme (funder number N/A). The contribution of RET to this work was supported by a National Health and Medical Research Council (NHMRC) Programme Grant (APP1113532).

Competing interests All authors declare: no support from any organisation for the submitted work; no financial relationships with any organisations that might have an interest in the submitted work; no other relationships or activities that could appear to have influenced the submitted work.

\section{Patient consent for publication Not required.}

Ethics approval All recruitment and data collection procedures were approved by the Sydney Local Health District Human Research Ethics Committee (Reference number: X20-0023).

Provenance and peer review Not commissioned; externally peer reviewed.

Data availability statement Data are available on reasonable request. All data relevant to the study are available on reasonable request to the corresponding author.

Supplemental material This content has been supplied by the author(s). It has not been vetted by BMJ Publishing Group Limited (BMJ) and may not have been peer-reviewed. Any opinions or recommendations discussed are solely those of the author(s) and are not endorsed by BMJ. BMJ disclaims all liability and responsibility arising from any reliance placed on the content. Where the content includes any translated material, BMJ does not warrant the accuracy and reliability of the translations (including but not limited to local regulations, clinical guidelines, terminology, drug names and drug dosages), and is not responsible for any error and/or omissions arising from translation and adaptation or otherwise.

Open access This is an open access article distributed in accordance with the Creative Commons Attribution Non Commercial (CC BY-NC 4.0) license, which permits others to distribute, remix, adapt, build upon this work non-commercially, and license their derivative works on different terms, provided the original work is properly cited, appropriate credit is given, any changes made indicated, and the use is non-commercial. See: http://creativecommons.org/licenses/by-nc/4.0/.

\section{ORCID iDs}

Joshua Zadro http://orcid.org/0000-0001-8981-2125

Denise A 0'Connor http://orcid.org/0000-0002-6836-122X

Kirsten McCaffery http://orcid.org/0000-0003-2696-5006

Sascha Karunaratne http://orcid.org/0000-0001-7518-0307

Min Jiat Teng http://orcid.org/0000-0003-4948-0335

\section{REFERENCES}

1 Medicare item reports 2000/2001 to 2018/19. Available: http:// medicarestatistics.humanservices.gov.au/statistics/mbs_item.jsp [Accessed 01 Jun 2020].

2 Judge A, Murphy RJ, Maxwell R, et al. Temporal trends and geographical variation in the use of subacromial decompression and rotator cuff repair of the shoulder in England. Bone Joint J 2014;96B:70-4.

3 Paloneva J, Lepola V, Äärimaa V, et al. Increasing incidence of rotator cuff repairs--a nationwide registry study in Finland. BMC Musculoskelet Disord 2015;16:189.
4 Zhang AL, Montgomery SR, Ngo SS, et al. Analysis of rotator cuff repair trends in a large private insurance population. Arthroscopy 2013;29:623-9.

5 Karjalainen TV, Jain NB, Page CM, et al. Subacromial decompression surgery for rotator cuff disease. Cochrane Database Syst Rev 2019;1:CD005619.

6 Karjalainen TV, Jain NB, Heikkinen J, et al. Surgery for rotator cuff tears. Cochrane Database Syst Rev 2019;12:CD013502.

7 Jones T, Carr AJ, Beard D, et al. Longitudinal study of use and cost of subacromial decompression surgery: the need for effective evaluation of surgical procedures to prevent overtreatment and wasted resources. BMJ Open 2019;9:e030229-e.

8 Stacey D, Légaré F, Lewis K, et al. Decision AIDS for people facing health treatment or screening decisions. Cochrane Database Syst Rev 2017;4:CD001431.

9 Coulter A, Stilwell D, Kryworuchko J, et al. A systematic development process for patient decision AIDS. BMC Med Inform Decis Mak 2013;13 Suppl 2:S2.

10 Elwyn G, O'Connor A, Stacey D, et al. Developing a quality criteria framework for patient decision AIDS: online international Delphi consensus process. BMJ 2006;333:417.

11 Coxeter PD, Mar CD, Hoffmann TC. Parents' expectations and experiences of antibiotics for acute respiratory infections in primary care. Ann Fam Med 2017;15:149-54.

12 O'Connor D, Hoffmann T, McCaffery K. 85 evaluating a patient decision aid for people with degenerative knee disease considering arthroscopic surgery: protocol for a randomised controlled trial. BMJ Evid Based Med 2019;24:A48-A.

13 Abhyankar P, Volk RJ, Blumenthal-Barby J, et al. Balancing the presentation of information and options in patient decision AIDS: an updated review. BMC Med Inform Decis Mak 2013;13.

14 Carling CLL, Kristoffersen DT, Flottorp S, et al. The effect of alternative graphical displays used to present the benefits of antibiotics for sore throat on decisions about whether to seek treatment: a randomized trial. PLoS Med 2009;6:e1000140-e.

15 Montori VM, LeBlanc A, Buchholz A, et al. Basing information on comprehensive, critically appraised, and up-to-date syntheses of the scientific evidence: a quality dimension of the International patient decision aid standards. BMC Med Inform Decis Mak 2013;13 Suppl 2:S5.

16 Santesso N, Rader T, Nilsen ES, et al. A summary to communicate evidence from systematic reviews to the public improved understanding and accessibility of information: a randomized controlled trial. J Clin Epidemiol 2015;68:182-90.

17 Trevena LJ, Zikmund-Fisher BJ, Edwards A, et al. Presenting quantitative information about decision outcomes: a risk communication primer for patient decision aid developers. BMC Med Inform Decis Mak 2013;13 Suppl 2:S7.

18 Tong A, Sainsbury P, Craig J. Consolidated criteria for reporting qualitative research (COREQ): a 32-item checklist for interviews and focus groups. Int J Qual Health Care 2007;19:349-57.

19 Clarke V, Braun V, Hayfield N. Thematic analysis. In: Qualitative psychology: a practical guide to research methods, 2015: 222-48.

20 Microsoft Corporation. Microsoft excel [Internet], 2018. Available: https://office.microsoft.com/excel

21 Joseph-Williams N, Newcombe R, Politi M, et al. Toward minimum standards for certifying patient decision AIDS: a modified delphi consensus process. Med Decis Making 2014;34:699-710.

22 Witteman HO, Vaisson G, Provencher T, et al. An 11-item measure of user- and human-centered design for personal health tools (UCD-11): development and validation. J Med Internet Res 2021;23:e15032.

23 Buchbinder R, Green S, Youd JM. Corticosteroid injections for shoulder pain. Cochrane Database Syst Rev 2003;1:CD004016.

24 Page MJ, Green S, McBain B. Manual therapy and exercise for rotator cuff disease. Cochrane Database Syst Rev 2016;6:CD012224.

25 Page MJ, Green S, Mrocki MA, et al. Electrotherapy modalities for rotator cuff disease. Cochrane Database Syst Rev 2016;6:CD012225.

26 Schwartz LM, Woloshin S, Welch HG. Using a drug facts box to communicate drug benefits and harms: two randomized trials. Ann Intern Med 2009;150:516-27.

27 Waldron C-A, van der Weijden T, Ludt S, et al. What are effective strategies to communicate cardiovascular risk information to patients? a systematic review. Patient Educ Couns 2011;82:169-81.

28 Zadro JR, Traeger AC, Décary S. Problem with patient decision AIDS BMJ Evid Based Med 2020. [Epub ahead of print: 21 May 2020]. doi:10.1136/bmjebm-2020-111371

29 O'Connor AM, Cranney A. User manual - acceptability, 1996. Available: www.ohri.ca/decisionaid 